\title{
SIMMEL E A CONFIANÇA
}

\author{
Simmel and Trust
}

Rodrigo Mota'

\section{Resumo}

Este artigo é uma breve exposição de uma das diversas manifestações do pioneirismo de Georg Simmel na sociologia: o estudo do conceito de confiança. Para isso, recorro aos pequenos trechos das obras nos quais o autor faz referência direta ao termo, inserindo-o no contexto das suas influências filosóficas e teoria social. Simmel percebeu como a impessoalidade crescente na modernidade fez a confiança tornar-se indispensável, como um mecanismo que torna a interação social possível dentro de um mundo no qual pouco se sabe sobre as outras pessoas. Recorre-se à própria história pessoal, ao mínimo conhecimento que se pode extrair sobre o outro e ao elemento que faz o tratamento simmeliano deste tema inovador: a suspensão, um ato de fé no próximo. Concluo reforçando a importância do estudo de Simmel sobre a confiança e de como a influência da Lebenssoziologie foi fundamental para fornecer a singularidade da sua visão, ao ver a vida social humana além da racionalidade instrumental.

Palavras-chave: Georg Simmel; Confiança; Vitalismo.

\begin{abstract}
This article is a brief exposition of one of the many manifestations of Georg Simmel pioneering in the field of sociology: the study of the concept of trust. For that, I resort to the small parts of the works on which the author directly refers to the term, inserting it in the context of his philosophical influences and social theory. Simmel realized how the growing impersonality of modernity has made trust even more necessary as a mechanism that makes social interaction possible in a world at which little is known about other people. One resorts to his own personal history, to the minimum knowledge that one can extract about the other and to the element that makes the simmelian treatment of this theme innovative: the suspension, an act of faith on the other. I conclude reinforcing the importance of Simmel's study on trust and how the influence of the Lebenssoziologie was fundamental to provide him with his singular view, by seeing human social life beyond instrumental rationality.
\end{abstract}

Keyword: Georg Simmel; Trust; Vitalism.

\footnotetext{
1 Programa de Pós-Graduação em Sociologia da Universidade Federal de Pernambuco (PPGS/UFPE). Email: rodrigomoin@gmail.com
} 
"Wird die menschliche Vergesellschaftung2 durch das Sprechenkönnen bedingt, so wird sie [...] durch das Schweigenkönnen geformt'3 (Georg Simmel, 1992 [1908]).

Georg Simmel (1858-1918) é hoje considerado um dos pais fundadores da sociologia. Apesar das suas contribuições fundamentais a essa disciplina, Simmel se considerava antes de tudo um filósofo (VANDENBERGHE, 2005). Por essa razão se torna fundamental, para entendermos sua sociologia, que compreendamos, antes de tudo, o essencial de suas raízes filosóficas. Sem dúvida, seus pressupostos giram em torno da filosofia de Immanuel Kant, tendo Simmel inclusive defendido sua tese de doutoramento sobre este autor. Posteriormente, Simmel faria parte do movimento filosófico conhecido como Neokantismo, mais especificamente da escola conhecida hoje como “escola de Baden” (VANDENBERGHE, 2005, p. 39).

A outra grande influência para a filosofia de Simmel é a corrente filosófica denominada Lebensphilosophie, ou Vitalismo ${ }^{4}$, que alcançou sua maturidade com o filósofo Henri Bergson na França. Contudo, "diferentemente do vitalismo francês, a filosofia da vida de Simmel é inspirada em Schopenhauer e Nietzsche" (VANDENBERGHE, 2005, p. 48), apesar da inegável influência e proximidade do sociólogo alemão com o filósofo francês, sendo Simmel apelidado por alguns da sua época de "o Bergson alemão" (BISTIS, 2005, p. 396). Bergson pode ser considerado "o fundador francês de uma verdadeira teoria da vida” (DELITZ, 2015, p. 44) e Simmel chegou a afirmar, num artigo sobre o filósofo francês, que o conceito de vida é o terceiro estágio da filosofia ocidental, a qual passou pela ideia de substância nos gregos e as formas mecânicas na filosofia moderna (BLEICHER, 2007; SIMMEL, 1922).

\footnotetext{
2 De acordo com Kurt Wolff (1950, p. LXIII), "the key term 'Vergesellschaftung' [...] has consistently been translated as 'sociation" [o termo chave 'Vergesellschaftung' [...] tem sido consistentemente traduzido como 'sociação'].

3 "Se a sociação humana é condicionada pela capacidade de falar, então ela será formada pela capacidade de silenciar" (SIMMEL, 1992, p. 426).

4 Esta corrente de pensamento, cujas origens remontam aos séculos XVIII e XIX (FRASER et al, 2005, p. 01), surge primeiramente como um contraponto ao mecanicismo de Isaac Newton e Immanuel Kant, chegando à concepção da realidade como um devir contínuo e a vida (daí o termo vitalismo) como a manifestação por excelência deste devir. As origens da Lebensphilosophie remontam a Johann Wolfgang von Goethe, passando por Arthur Schopenhauer e Friedrich Nietzsche.
} 
De fato, Simmel entendia a "vida em termos de vida social. Esta é a originalidade do seu vitalismo. [...] Para Simmel, vida social é literalmente vida social. Este é o vitalismo sociológico, a Lebenssoziologie" (LASH, 2005, p. 10). Resumindo essas influências filosóficas, Frédéric Vandenberghe define as três fases distintas do pensamento deste autor da seguinte forma:

em uma primeira fase (1879-1900), que se encerra com seu livro sobre a diferenciação social, ele é influenciado pelo neodarwinismo de Spencer e filosofa como positivista [...]; na segunda fase (19011908), que começa com seu livro sobre a filosofia da história, rompe com o positivismo e se aproxima da escola neokantiana de Baden; por fim, perto do final da vida (1906-1918), sofre a influência de Bergson e se torna vitalista, de que é exemplo Lebensanschauung (Visão do mundo), seu testamento filosófico (VANDENBERGHE, 2005, p. 39).

Analiso neste artigo a contribuição original desse clássico da sociologia que é Georg Simmel ao estudo da confiança. Apesar de aparecer em poucas páginas de suas obras, essa contribuição está subentendida em diversas temáticas tratadas por esse autor. Em particular, o elemento de suspensão da falta de conhecimento "suficiente" sobre o outro na interação baseada em confiança é o componente que mais chama a atenção na análise simmeliana sobre o tema, e do qual trataremos com a ajuda de uma interpretação de um autor contemporâneo. Nos casos específicos tratados aqui, mostramos as contribuições diretas presentes nos seus textos sobre a metrópole, o dinheiro e sobre o segredo e a sociedade secreta. Finalizo o artigo ressaltando os aspectos positivos de sua Lebenssoziologie.

\section{Breve introdução à teoria social simmeliana}

O pensamento de Simmel não é sistemático, mas possui um método. Ele consistia em observar a realidade não como um todo, mas através de pequenas partes, pois "como não dispõe de um sistema que englobe a totalidade do mundo - a totalidade não é dada como ponto de partida, mas adquirida por 'errâncias' analógicas como ponto de chegada" (VANDENBERGHE, 2005, p. 42). Por isso, ele muda frequentemente tanto de objeto, quanto de ponto de vista. Disso se deriva que "o real só pode ser apreendido através de uma pluralidade de perspectivas que captam, cada 
uma, um aspecto da vida sem jamais esgotar sua significação" (VANDENBERGHE, 2005, p. 45).

A melhor forma encontrada por ele, derivada de suas influências filosóficas, para esta observação da vida social, foi o desenvolvimento "do (meta-) conceito do dualismo, da dualidade em interação ou da dialética sem sintese” (VANDENBERGHE, 2005, p. 52). Diversas análises feitas por Simmel constituem no estudo de oposições, como, por exemplo, a relação distinção-imitação presente no seu ensaio sobre a Moda, mas a relação dualista fundamental está na dualidade da oposição dos conteúdos e das formas, que juntamente com o conceito "da interação ou da ação em reciprocidade [...] [que] indica que tudo está ligado a tudo e que tudo se confunde, [...] constitui totalmente o alicerce sobre o qual é construída a obra de Simmel" (VANDENBERGHE, 2005, p. 58-59).

Essas interações seriam o que formaria de fato o que entendemos como sociedade, já que não se pode pensar o indivíduo sozinho ou a sociedade desprovida de indivíduos como real. Portanto, sociedade seria definida como "um número de indivíduos conectados por interação"5 (WOLFF, 1950, p. XXX). Simmel, então, abstraía elementos da realidade histórica e os recombinava para seus estudos específicos no que ele chamava de sociologia "pura" ou "formal”, que consistia na investigação "das próprias formas sociais ${ }^{6}$ que fazem a 'sociedade (e sociedades) além da mera soma dos humanos vivos"7 (WOLFF, 1950, p. XXXIV). Essa sociologia se diferencia daquela denominada por ele de sociologia filosófica, que estudaria as bases epistemológicas e as bases das formas e normas da sociedade. Além dessas, ainda existia outra sociologia, chamada "sociologia geral", que analisaria a história, mas com um olhar particular sociológico, segundo Simmel (apud WOLFF, 1950, p. XXXIII) seria “o estudo 'do todo da vida

\footnotetext{
5 Tradução minha de "a number of individuals connected by interaction" (WOLFF, 1950, p. xxx).

6 Exemplos dessas formas, de acordo com Kurt Wolff (1950, p. XXXIV), são "superiority and subordination, competition, division of labor, formation of parties, representation, inner solidarity coupled with exclusiveness toward the outside [superioridade e subordinação, competição, divisão do trabalho, formação de partidos, representação, solidaridade interna acoplada com exclusividade em relação ao exterior]".

7 Tradução minha de "the societal forms themselves, which make society (and societies) out of the mere sum of living men" (1950, p. XXXIV).
} 
histórica à medida que este é formado societalmente"8. Essas breves considerações sobre a filosofia e as sociologias de Georg Simmel são fundamentais para se compreender a forma que ele trata os seus objetos, no caso deste trabalho em particular, a confiança. Um aspecto fundamental da teorização de Simmel sobre a confiança é o seu papel de facilitadora da ação, principalmente num mundo com excesso de informações como é o caso desde o advento da modernidade, na qual não se pode conhecer tudo com suficiente profundidade, nem tampouco ficar paralisado na inação. É sobre este ponto que escreveremos a seguir.

\section{A confiança entre a certeza e a ignorância}

Apesar de tratar do tema da confiança em apenas algumas passagens de duas de suas obras, "uma no Philosophiedes Geldes [1900] e duas no Soziologie [1908], se estendendo a não mais do que dez páginas ao todo" (MÖLLERING, 2001, p. 405), Simmel foi o primeiro, nas ciências sociais, a abordar a confiança. Para se entender a importância dada por Simmel à confiança, basta observar a relação entre o fato social moderno sobre a qual esta apareceu pela primeira vez nos seus trabalhos, a saber, a "economia de crédito" (Kreditwirtschaft), e a importância desse fato para a modernidade, que, segundo Simmel (1992, p. 389) “é uma 'economia de crédito' em um sentido mais além do que o econômico"9. Ele chama a atenção ainda para "como, sem a crença dos homens uns nos outros, a sociedade ruiria"10 e que "esse tipo de crença não é um conhecimento indutivo enfraquecido [grifo meu]. [...] no caso do crédito, a confiança em alguém aparece como um desses momentos difíceis de descrever, que é caracterizado mais puramente na fé religiosa"11 (SIMMEL, 1989, p. 216).

\footnotetext{
8 Tradução minha de "the study "of the whole of historical life insofar as it is formed societally" (1950, p. XXXIII).

9 Tradução minha de "das in einem viel weiteren als dem ökonomischen Sinne ,Kreditwirtschaft' ist" (SIMMEL, 1992, p. 389).

10 Tradução minha de "Wie ohne den Glauben der Mensch aneinander überhaupt die Gesellschaft auseinanderfallen würde”. (SIMMEL, 1989, p. 215).

11 Tradução minha de "Diese Art des Glaubens ist nichts als ein abgeschwächtes induktives Wissen. [...] in dem Fall des Kredites, des Vertrauen auf jemanden, kommt zu diesem noch weiteres, schwer zu beschreibendes Moment hinzu, das am reinsten in dem religiösen Glauben verkörpert ist" (SIMMEL, 1989, p. 216).
} 
A necessidade da confiança é ampliada na modernidade graças ao fenômeno das grandes cidades: das Metrópoles. A vida rural é mais lenta, mais habitual e com relacionamentos mais emocionais. Na metrópole há a exigência de uma maior consciência, se "reage com a cabeça ao invés do coração. [...] [n]uma atentividade aumentada e uma predominância de inteligência12" (SIMMEL, 1950, p. 410). O uso do intelecto é o que ajuda a preservar a subjetividade diante do poder das forças sociais. A economia monetária somada à grande quantidade de estímulos nervosos presentes nas grandes cidades faz com que as pessoas reajam com desconfiança natural à vida metropolitana, gerada pelo tipo de relação objetiva que ela reforça. É por isso que os metropolitanos são vistos como "frios" pelos indivíduos das cidades pequenas. Esta reserva diante dos próximos também se manifesta em repulsa e estranhamento.

Vive-se numa hierarquia de simpatias e antipatias, segundo Simmel (1950). A antipatia nos protege tanto da indiferença, por já ser uma tomada de posição frente ao outro, quanto do fluxo indiscriminado de novas sugestões. Já é um pré-conceito, servindo como um filtro frente ao fluxo contínuo de informações novas. Dinheiro, metrópole e intelecto estão conectados. Todos têm uma atitude fatual com coisas e pessoas, um posicionamento objetivo. A pessoa intelectualmente sofisticada, segundo Simmel, é indiferente diante da genuína individualidade e subjetividade, pois estas não se encaixam nas operações puramente lógicas. O dinheiro, como o intelecto, regula tudo pelo que há de comum, reduz qualidades individuais à questão de "quanto custam?". O dinheiro, relacionado a questões objetivas, não de valor (no sentido moral), deixa, por isso, o egoísmo tanto do consumidor quanto do produtor correr mais solto.

Simmel se aprofunda na temática da confiança no capítulo "O segredo e a sociedade secreta" (Das Geheimnis und die geheime Gesellschaft) do seu livro "Sociologia: Investigação sobre as formas de sociação" (Soziologie: Untersuchungenüber die Formen der Vergesellschaftung), de 1908. A aproximação do autor a este tema se dá a partir da ideia presente na frase

12 Tradução minha de "reacts with his head instead of his heart [...] a heightened awareness and a predominance of intelligence". (SIMMEL, 1950, p. 410). 
pela qual o capítulo é iniciado: "Todos os relacionamentos dos homens uns com os outros depende evidentemente de que eles saibam algo uns dos outros"13 (SIMMEL, 1992, p. 383). Isto posto, Simmel analisa diversas formas de interação entre os seres humanos e sua relação com o conhecimento mútuo entre os indivíduos. Como não se pode conhecer o outro em absoluto, os indivíduos formam, a partir do conhecimento acessivel a eles, uma espécie de conteúdo pessoal sobre a personalidade desse outro.

A forma desse "conteúdo pessoal" é algo bastante subjetivo, fazendo com que a impressão que um indivíduo $\mathrm{A}$ tem de um indivíduo $\mathrm{M}$ seja distinta da que um indivíduo $\mathrm{B}$ tem deste mesmo indivíduo, não significando, entretanto, que uma esteja mais correta do que a outra (SIMMEL, 1992). A interpretação dos dados sobre M por A depende das particularidades da personalidade de A, do relacionamento particular deste com $\mathrm{M}$, além, claro, da sorte atuando sobre a vida e, consequentemente, sobre o relacionamento de ambos.

Neste processo, um indivíduo fornece determinadas informações sobre si a outro enquanto o outro faz o mesmo, formando em cada um uma ideia do outro; assim, essa troca de informações funciona como auxiliar no momento em que um dos indivíduos deseja realizar alguma ação, inclusive a seleção das novas informações a serem repassadas. Nas palavras de Simmel (1992, p. 385), "nossas relações se desenvolvem através das bases de um conhecimento mútuo um do outro e esse conhecimento através das bases das efetivas relações" ${ }^{14}$. Neste processo, estão presentes dois tipos de conhecimentos, o do Ser ("quem se é", conhecimento de si próprio, experienciado unicamente em sua totalidade pelo próprio indivíduo) e da representação do Ser (conhecimento gerado pelo outro a partir das informações previamente selecionadas e repassadas pelo "sujeito agora objetificado"). Nesta troca de informações, o sujeito ao mesmo tempo se mostra e se oculta, se revela e engana, por essa razão, "nenhum outro objeto pode, através de si próprio, nos informar sobre si ou se esconder, como o ser

13 Tradução minha de "Alle Beziehung von Menschen untereinander ruhen selbstverständlich darauf, daß sie etwas von einander wissen" (SIMMEL, 1992, p. 383).

14 Tradução minha de "So entwickeln unsere Verhältnisse auf der Basis eines gegenseitigen Wissens von einander und dieses Wissen auf der Basis der tatsächlichen Verhältnisse" (SIMMEL, 1992, p. 385). 
humano pode, pois nenhum outro modifica seu comportamento levando em consideração o seu tornar-se conhecido"15 (SIMMEL, 1992, p. 386, [grifo meu]).

A partir dessas considerações, Simmel observa como a mentira é um fenômeno importantíssimo para a sociologia. Nas sociedades primitivas, os indivíduos pelas próprias condições em que viviam - a curta duração da vida, a maior proximidade em relação aos outros indivíduos e a necessidade da cooperação direta com estes - limitavam seus interesses mentais às experiências imediatas ou a tradições recebidas, gerando as diversas superstições e equívocos (do ponto de vista europeu) presentes na cultura destes povos (SIMMEL, 1992). Em contraste com isso, "nas mais ricas e adiantadas culturas, a vida é formada por milhares de pressuposições, as quais o indivíduo não pode de forma alguma acompanhar e verificar até sua origem, mas devem aceitar por lealdade ou crença"16 (SIMMEL, 1992, p. 389).

É a partir dessa crença que Simmel vai desenvolver a sua ideia de confiança, focalizando sua especial importância nas sociedades modernas. Segundo Simmel, "nossa existência moderna jaz [...] na crença na boa-fé dos outros"17. Ele ainda retorna ao comentário feito anteriormente na sua Philosophie des Geldes - que a confiança é uma das forças sintéticas mais importantes da sociedade - e coloca a confiança como "a hipótese da conduta futura, que é segura o suficiente para fundamentar a ação prática"18 e - num dos mais importantes insights presentes numa nota de rodapé- a confiança é apresentada como a "hipótese de uma situação intermediária entre saber e não-saber em torno dos seres humanos. Aquele que conhece completamente não precisa confiar, aquele que não conhece

\footnotetext{
15 Tradução minha de "Kein andres Objekt kann uns in dieser Weise von sich aus aufklären oder sich verstecken, wie der Mensch es kann, weil kein andres sein Verhalten durch die Rücksicht auf sein Erkanntwerden modifiziert" (SIMMEL, 1992, p. 386).

16 Tradução minha de "Bei reicherem und weiterem Kulturleben dagegen steht das Leben auf tausend Voraussetzungen, die der Einzelne überhaupt nicht bis zu ihrem Grunde verfolgen und verifizieren kann, sondern die er auf Treu und Glauben hinnehmen muß” (SIMMEL, 1992, p. 389).

17 Tradução minha de "ruht unsre moderne Existenz [...] auf dem Glauben an die Ehrlichkeit des andern" (SIMMEL, 1992, p. 389).

18 Tradução minha de "die Hypothese künftigen Verhaltens, die sicher genug ist, um praktisches Handeln darauf zu gründen" (SIMMEL, 1992, p. 393).
} 
nada não pode racionalmente confiar"19 (SIMMEL, 1992, p. 393). A quantidade de conhecimento e desconhecimento nas relações de confiança é relativa, variando mais para um lado ou para outro, dependendo da natureza do relacionamento. Por exemplo, um relacionamento comercial, mediado pelo dinheiro, é impessoal e por isso essencialmente não requer conhecimento particular entre as partes, diferente de relacionamentos mais próximos. Usando o exemplo fornecido por Simmel, veremos que

o comerciante que vende à outro cereal ou petróleo só necessita saber se a quantia é suficiente; quando ele, no entanto, desejar tomar um outro como sócio, ele deverá conhecer não apenas a fortuna ou qualidades gerais deste, mas sim conhecer amplamente sua personalidade, deve conhecer sua decência, sua compatibilidade, seu temperamento ousado ou vacilante; e sobre tal - mútuo conhecimento jaz não somente o comprometimento da relação, mas sua total continuidade, as ações diárias comuns, a divisão funcional entre os companheiros. O segredo da personalidade está agora sociologicamente limitado (SIMMEL, 1992, p. 394-395) ${ }^{20}$.

Obviamente, só se pode conhecer sobre o outro aquilo que este nos mostra. A definição do limite do conhecimento mútuo, a chamada discrição (que também inclui a limitação do poder concedido pelo confiador ao confiado), é posta por Simmel (1992) na forma de um imperativo derivado do imperativo “o que não é proibido, é permitido, e: o que não é permitido, é proibido"21, que neste caso se torna: "o que não é ocultado, pode ser conhecido, e: o que não é manifestado, pode igualmente não ser conhecido"22 (SIMMEL, 1992, p. 396). Para este autor, é como se espécies de "esferas ideais" rodeassem as personalidades dos indivíduos, limitando o que é segredo e o que deve ser exposto sobre estas, nas quais não se poderia penetrar sem destruir o valor das mesmas personalidades.

\footnotetext{
19 Tradução minha de "Hypothese ein mittlerer Zustand zwischen Wissen und Nichtwissen um den Menschen. Dervöllig Wissende braucht nicht zu vertrauen, der völlig Nichtwissende kann vernünftigerweise nicht einmal vertrauen" (SIMMEL, 1992, p. 393).

20 Tradução minha de "Der Kaufmann, der einem andren Getreide oder Petroleum verkauft, braucht nur zu wissen, ob dieser für den Betreg gut ist; sobald er aber einen andren zum Associé nimmt, muß er nicht nur die Vermögenslage und gewisse ganz allgemeine Qualitäten desselben kennen, sondern muß ihn als Persönlichkeit weitgehend durchschauen, muß seine Anständigkeit, seine Verträglichkeit, sein wagendes oder zagendes Temperament kennen; und auf solcher - gegenseitiger - Kenntnis ruht nicht nur das Eingehen der Verhältnisses, sondern seine gesamte Fortsetzung, die täglichen gemeinsamen Aktionen, die Funktionsteilung unter den Kompagnons. Das Geheimnis der Persönlichkeit ist jetzt soziologisch beschränkter” (SIMMEL, 1992, p. 394-395).

21 Tradução minha de "was nicht verboten ist, ist erlaubt, und: was nicht erlaubt ist, ist verboten" (SIMMEL, 1992, p. 396).

22 Tradução minha de "was nicht verborgen wird, darf gewußt werden, und: was nicht offenbart wird, darf auch nichtgewußt werden" (SIMMEL, 1992, p. 396).
} 
Pensando as sociedades secretas, Simmel se dá conta da forma especial de confiança que estas associações podem nos apresentar pelo seu caráter de protetoras dos seus membros, fazendo-os "invisiveis". Este tipo de confiança difere da confiança no nivel estritamente individual. Um indivíduo pode ocultar certos aspectos da sua existência, mas não a sua existência em si, enquanto que a sociedade secreta, como entidade, pode manter sua existência oculta do restante da sociedade convencional (SIMMEL, 1992, p. 422). Este tipo de sociedade secreta referida por Simmel difere, por exemplo, da Maçonaria, que é publicamente conhecida, mantendo apenas seus rituais em segredo (SIMMEL, 1992).

A confiança entre os membros de uma sociedade secreta se fundamenta principalmente na capacidade dos seus membros de manter silêncio, algo análogo às "esferas ideais" referidas anteriormente, mas em um nivel supra-individual, onde o que conta não é a razão ou as emoções de um indivíduo em relação a sua personalidade, mas a razão e as emoções de diversos indivíduos interagindo em nome de seus segredos mútuos, inclusive o da própria existência do grupo. Esta confiança tem de ser constantemente renovada para a própria continuidade da sociedade secreta, pois a confiança pode, a qualquer momento, ser quebrada através da revelação dos segredos, a qual pode surgir por simples sinais descuidados de um dos membros (SIMMEL, 1992, p. 425). Por essa razão, Simmel considerou a sociedade secreta como exemplar e uma espécie de "tipo ideal" weberiano ${ }^{23}$ do que seria a manifestação da confiança entre indivíduos. Nas palavras do próprio autor:

A sociedade secreta opera [...] um adestramento altamente efetivo da solidariedade moral entre os homens. Na confiança dos seres humanos nos outros jaz um valor moral tão alto, como no caso em que esta confiança é correspondida; sim, talvez um ainda mais livre e meritório, já que uma confiança que nos é concedida contém quase um poder compulsório, e enganá-la exige uma maldade totalmente positiva. A confiança, pelo contrário, se "oferece"; ela não pode ser

\footnotetext{
23 Um tipo ideal é formado pela acentuação unilateral de um ou mais pontos de vista e pela sintese de diversos fenômenos individuais concretos difusos, discretos, mais ou menos presentes e, às vezes, ausentes, que são organizados de acordo com aqueles pontos de vista enfatizados unilateralmente, em um construto analítico unilateral. [Tradução minha de: "An ideal type is formed by the one-sided accentuation of one or more points of view and by the synthesis of a great many diffuse, discrete, more or less present and occasionally absent concrete individual phenomena, which are arranged according to those one-sidedly emphasized viewpoints into a unified analytical construct"] (WEBER, 1949, p. 90).
} 
exigida na mesma medida em que se corresponde a ela quando é oferecida $^{24}$ (SIMMEL, 1992, p. 425 [grifo meu]).

No início da sua discussão sobre a confiança, Simmel alerta, na extensa nota de rodapé já mencionada, que existe um outro tipo de confiança para além da dicotomia conhecer/desconhecer, que seria "o que se chama de fé dos seres humanos nos outros e que pertence à categoria da fé religiosa"25 (SIMMEL, 1992, p. 393). Da mesma forma que se tem fé em Deus sem necessariamente possuir provas empíricas justificáveis suficientes, também se acredita em outro indivíduo sem que essa fé seja realmente justificável através do merecimento da pessoa, às vezes até à revelia de alertas sobre o contrário. "Essa confiança [...] não é mediada nem por experiências nem por hipóteses, mas através de um comportamento primário da alma em relação ao outro"26 (SIMMEL, 1992, p. 393).

Esse elemento se encontra em sua forma pura na religião. $\mathrm{Na}$ confiança, e, consequentemente, nas ações baseadas nela, ele serve como um estímulo ao conhecimento ou suspeita já presente na interação. Simmel não se estende muito na discussão deste elemento, mas, pelas suas palavras, percebe-se que ele o trata como muito importante para o estudo não só da confiança, mas também das relações humanas em geral. Isto se percebe quando o autor alemão considera que seria "uma categoria básica do comportamento humano, retornando ao sentido metafísico das nossas relações e que se realiza apenas empiricamente, casualmente, fragmentariamente, através das razões conscientes básicas e singulares da confiança”27 (SIMMEL, 1992, p. 393-394).

\footnotetext{
24 Tradução minha de "bewirken die geheimen Gesellschaften [...] eine höchst wirkungsvolle Schulung des moralischen Verbundenseins unter den Menschen. Denn in dem Vertrauen des Menschen auf den andern liegt ein ebenso hoher moralischer Wert, wie darin, daß diesem Vertrauen entsprochen wird; ja, vielleicht ein noch freierer und verdienstvollerer, da ein Vertrauen, das uns gewährt ist, ein fast zwingendes Präjudiz enthält, und es zu täuschen schon eine ganz positive Schlechtigkeit fordert. Das Vertrauen dagegen ,schenkt' man; es kann nicht in demselben Maße verlangt werden, wie daß man ihm entspreche, wenn es einmal geschenkt ist" (SIMMEL, 1992, p. 425).

25 Tradução minha de "den man den Glauben des Menschen an einen andern nennt und der in die Kategorie des religiösen Glaubens gehört” (SIMMEL, 1992, p. 393).

26 Tradução minha de "Dieses Vertrauen [...] ist weder durch Erfahrungen noch durch Hypothesen vermittelt, sondern ein primäres Verhalten der Seele in Bezug auf den andern” (SIMMEL, 1992, p. 393).

27 Tradução minha de "eine Grundkategorie menschlichen Verhaltens, auf den metaphysischen Sinn unserer Beziehungen zurückgehend und durch die bewußte, singulären Gründe des Vertrauens nur empirisch, zufällig, fragmentarisch verwirklicht” (SIMMEL, 1992, p. 393-394).
} 
No ano de 2001, foi publicado na revista Sociology (vol. 35, $\mathrm{n}^{\circ}$ 2) um artigo do cientista social alemão Guido Möllering sobre a confiança intitulado "The Nature of Trust: From Georg Simmel to a Theory of Expectation, Interpretation and Suspension", no qual chamava os pesquisadores a retornarem a Simmel nos estudos sobre a confiança e construía, o próprio Möllering, uma teoria sobre este tema, baseando-se nos estudos do pensador alemão. Para Möllering "confiança pode ser imaginada como o processo mental de saltar - permitido pela suspensão - através do desfiladeiro do desconhecivel da terra da interpretação para dentro da terra da expectativa"28 (MÖLLERING, 2001, p. 412). Neste sentido, a confiança

(...) consiste de três elementos. O primeiro é expectativa: o estado alcançado ao final do processo da confiança, o qual pode ser tanto favorável (no caso da confiança) ou desfavorável (desconfiança). Em segundo lugar, a interpretação captura a ideia que o experienciar humano do mundo da vida fornece as bases para confiança ('boas razões'). No entanto, em terceiro lugar, o salto mental da confiança (da interpretação para a expectativa) precisa ser possibilitado pela suspensão: o suporte do desconhecivel que representa um aspecto definitivo da natureza da confiança ${ }^{29}$ (MÖLLERING, 2001, p. 417 [grifos do autor]).

Möllering argumenta que a maioria das pesquisas sobre a confiança consistem de estudos sobre a interpretação, assumindo que as "boas razões" produzirão confiança sem o salto promovido pela suspensão (MÖLLERING, 2001, p. 412). Ele dá ênfase, no referido artigo, a este último elemento, derivado da noção de confiança como uma espécie de fé oferecida por Simmel. Para Möllering, a confiança "representa um processo no qual atingimos um ponto onde nossas interpretações são aceitas e nossa consciência do desconhecido, desconhecível e não-resolvido é suspensa"30 (MÖLLERING, 2001, p. 414 [grifos do autor]).

\footnotetext{
28 Tradução minha de "trust can be imagined as the mental process of leaping - enabled by suspension - across the gorge of the unknowable from the land of interpretation into the land of expectation" (MÖLLERING, 2001, p. 412).

29 Tradução minha de "consists of three elements. The first is expectation: the state reached at the end of the trust process and which can be either favourable (in the case of trust) or unfavourable (distrust). Secondly, interpretation captures the idea that human experiencing of the life-world gives bases for trust ('good reasons'). However, thirdly, the mental leap of trust (from interpretation to expectation) needs to be enabled by suspension: the bracketing of the unknowable which represents a defining aspect of the nature of trust" (MÖLLERING, 2001, p. 417).

30 Tradução minha de "stands for a process in which we reach a point where our interpretations are accepted and our awareness of the unknown, unknowable and unresolved is suspended" (MÖLLERING, 2001, p. 414).
} 
Dessa maneira, suspensão se define como "o mecanismo que suporta nossa incerteza e ignorância, fazendo o conhecimento interpretativo momentaneamente 'certo', permitindo o salto favorável (ou desfavorável) para a expectativa"31 (MÖLLERING, 2001, p. 414), a expectativa, por sua vez, representa o output do processo da confiança e o input das suas consequências como, por exemplo, das ações de cooperação entre os indivíduos. A partir desses argumentos, Möllering considera fundamental este retorno aos estudos simmelianos sobre a confiança, fundamentalmente no seu aspecto menos evidente, mas fundamental, do componente de fé entre os seres humanos.

\section{Conclusão}

Nas poucas páginas que dedicou sobre a confiança, Simmel, como se pode observar, nos forneceu insights fascinantes e abrangentes sobre este fenômeno social, indo desde sua fundamentação cognitiva, aos seus aspectos morais e metafísicos. Como vimos nos comentários de Guido Möllering (2001) sobre estes estudos de Simmel, o aspecto mais inovador e intrigante descoberto pelo autor clássico alemão sobre a confiança, o salto de fé - a suspensão - permanece, ironicamente, como um dos mais negligenciados nos estudos posteriores sobre a confiança.

Talvez se os teóricos diminuíssem a importância do papel da racionalidade na vida social humana - diminuição efetuada em Simmel sem dúvida sob a influência da filosofia vitalista, que vem sendo revivida na última década graças às "mudanças nas ciências, com o surgimento da ideia de incerteza e complexidade e o surgimento da sociedade de informação global". E também "porque a noção de vida sempre favoreceu uma ideia de tornar-se ao invés de ser, de movimento sobre o estático, de ação sobre estrutura, de corrente e fluxo" (LASH, 2006, p. 323 [grifos do autor]).

Poder-se-ia até pensar que esta “irracionalidade" é na verdade bastante "racional", considerando que as consequências deste salto de fé permitem que a sociedade funcione minimamente, principalmente por não

\footnotetext{
31 Tradução minha de "the mechanism that brackets out uncertainty and ignorance, thus making interpretative knowledge momentarily 'certain' and enabling the leap to favourable (or unfavourable) expectation" (MÖLLERING, 2001, p. 414).
} 
exigir uma reflexividade constante. Aí jaz a importância da confiança simmeliana, em nos mostrar o quão contingente é a realidade social e o quanto de evidência sobre esta realidade se perde quando é analisada através de modelos deterministas. Com o aumento dos efeitos da modernidade, em particular das incertezas geradas pelo desconhecimento interpessoal - o que Talcott Parsons e Niklas Luhmann chamaram de duplacontingência (MOTA, 2016) - particularmente nas grandes metrópoles e sob a égide da economia monetária, a confiança se tornou uma possibilitadora essencial da ação social. Se esta leitura da confiança em Simmel tiver sido clara o suficiente, como era pretendido, acredito que sua importância ficará mais evidente ao mesmo tempo em que se perceba a onipresença da confiança/desconfiança na vida nas sociedades modernas.

\section{Referências}

BISTIS, Margo. Simmel and Bergson: the theorist and the exemplar of the 'blasé person'. Journal of European Studies, London, v. 35, n. 4, 2005.

BLEICHER, Josef. From Kant to Goethe: Georg Simmel on the way to Leben. Theory Culture Society, London, v. 24, n. 6, 2007.

DELITZ, Heike. Bergson-Effekte. Aversionen und Attraktionen im französischen soziologischen Denken. Weilerswist: Velbrück Wissenschaft, 2015.

FRASER, Mariam; KEMBER, Sarah; LURY, Celia. Inventive Life: Approaches to the New Vitalism. Special Issue: Theory, Culture and Society, v. 22, 2005.

LASH, Scott. Lebenssoziologie: Georg Simmel in the Information Age. Theory Culture Society, London, v. 22, n. 3, 2005.

. Life (Vitalism). Theory Culture Society, London: v. 23, n. 2-3, p. 323-349, 2006.

MÖLLERING, Guido. The nature of trust: from Georg Simmel to a theory of expectation, interpretation and suspension. Sociology, Durhan, v. 35, n. 2, 2001.

MOTA, Rodrigo. Confiança e complexidade social em Niklas Luhmann. PLURAL, Revista do Programa de Pós-Graduação em Sociologia da USP, São Paulo, v. 23, n. 2, 2016. 
SIMMEL, Georg. Philosophie des Geldes. Frankfurt am Main, Suhrkamp, 1989.

Soziologie: Untersuchungen über die Formen der Vergesellschaftung. Frankfurt am Main, Suhrkamp, 1992.

The Metropolis and Mental Life. In.: Kurt Wolff (ed.). The Sociology of Georg Simmel. Glencoe, Illinois: The Free Press, 1950.

1922.

Zur Philosophie der Kunst. Potsdam: Gustav Kiepenheuer Verlag,

VANDENBERGHE, Fréderic. As sociologias de Georg Simmel. Bauru, EDUSC/Belém, EDUPFA, 2005.

WEBER, Max. The Methodology of the Social Sciences. New York: Free Press, 1949.

WOLFF, Kurt. The Sociology of Georg Simmel. Glencoe, Illinois: The Free Press, 1950 